\title{
Microhabitat preferences in springs, as shown by a survey of nematode communities of Trentino (south-eastern Alps, Italy)
}

\author{
Aldo ZULLINI*, Fabio GATTI ${ }^{1)}$ and Roberto AMBROSINI \\ Department of Biotechnology \& Biosciences, University of Milano-Bicocca, Piazza d. Scienza 2, 20126 Milano, Italy \\ ${ }^{1)}$ Natural History Museum, via Farini 90, University of Parma, 43100 Parma, Italy \\ *e-mail corresponding author: aldo.zullini@unimib.it
}

\begin{abstract}
Ninety-four Alpine springs in Trentino, from 170 to $2792 \mathrm{~m}$ a.s.l., were studied and compared for their nematode communities. No nematode species appeared typical for Alpine springs (crenobionts or crenophiles); all the identified species were common in freshwater habitats, with a wide geographical range on a continental scale. The only notable, rare species was Eumonhystera tatrica Daday, 1896, a very small nematode that has apparently never been found since it was first described. Eighty springs with more than 7 specimens were retained for statistical analysis. Distinctness indices $\Delta^{+}$and $\Lambda^{+}$showed that only a few springs exceeded the funnel limits for such indices. The relationships between habitat features and community composition, and nematode ecology (c-p value, size, trophy) were investigated. The major abiotic factors influencing nematode community composition were water temperature and lithology (carbonate vs. crystalline). In addition, nematode communities from mosses differed from those sampled from other substrata in the same spring. The nematode-based Maturity Index increased with crenic water temperature, in contrast to other indices, such as Shannon diversity and Berger-Parker index, suggesting that r-strategist nematode species replace K-strategists along the temperature gradient. Lithology did not alter species richness, but the relative abundance of species present on carbonate and in non-carbonate substrata varied. The $4^{\text {th }}$-corner analysis showed significant correlations between temperature and species trophic group. In conclusion, nematodes are good ecological indicators of polluted vs unpolluted waters, but, at least in this case, cannot be used to differentiate between unpolluted habitats such as Alpine springs.
\end{abstract}

Key words: crenon, db-RDA, Eumonhystera tatrica, freshwater nematodes, bryophyte communities, Trentino

\section{INTRODUCTION}

Nematodes are one of the most abundant animal groups in freshwater sediments, where they can reach densities up to 11.4 million individuals per square meter (Michiels \& Traunspurger 2005). They play an important role in detritus food webs (Eyualem et al. 2001; Schmid \& Schmid-Araya 2002). It is possible to find several dozens of nematode species in a single body of water, lake or river (Traunspurger 2000), but it is difficult to estimate the total number of known freshwater species, because no clear distinction exists between freshwater and soil nematodes, or between freshwater and brackish water nematodes (Eyualem-Abebe et al. 2006). In a classic survey, Andrássy (1978) listed 605 freshwater (sensu lato) nematode species for Europe, 20 of them typical of springs (crenon). Spring habitats are a very restricted part of the global freshwater environment, but of crucial ecological importance (Cantonati et al. 2006). Crenic nematodes are an important component of the freshwater biota, but nematode studies are temporally and spatially scattered: Linstow (1901), Hoeppli (1926), Hoeppli \& Chu (1932), De Coninck (1935), Pax \& Soós (1943, 1951), Meyl (1953a, b, c, 1954), Paetzold (1958), Altherr (1969, 1974, 1976), Ocaña et al. (1986), Ocaña (1991a, b, 1992, 1993), Ocaña et al. (1992), Gagarin \& Lemzina (1992), Seiml-
Buchinger \& Traunspurger 2006. A total of 109 nematode species (terrestrial Tylenchomorpha excluded) have been identified, excluding those found only in thermal $\left(>30{ }^{\circ} \mathrm{C}\right)$ or saline $(>1100 \mu \mathrm{S})$ springs, which are not relevant to our research.

Springs can be considered ecotones between the hypogean and the epigean aquatic environment and, like caves, they are of special interest because of their typical rather constant abiotic parameters, e.g. temperature and chemical composition. Nematodes show adaptations to hypogeal habitats, lacking eyes and pigmentation, but they lack any particular adaptation to crenic habitats and, to the best of our knowledge, no nematode species is typical of springs (creonbionts or crenophiles). Some (e.g., Dorylaimida, Mononchida) are similar to troglobionts because of their long life cycle ( $K$-strategists) and are also well represented in the crenic habitat. In contrast, Rhabditina, being bacteriophagous and $r$-strategists, and requiring environments rich in organic matter, are not usually able to form dense, stable populations in springs. The Tylenchomorpha (Tylenchidae, Criconematidae, etc.) are sometimes found in some crenic sediments, but we consider them incidental, because they are phytoparasites and passively drained from the surrounding soil. Others, like the Mermithidae (arthropod parasites), can be brought to springs by their hosts, but in general all crenic nematodes are typical freshwater-inhabitants or, in some cases, also common in moist 
soil. In conclusion, nematodes live in springs mainly thanks to their high colonization ability, and it is likely that nematode communities living in these habitats reflect the rather stable, particular ecological situation. Given the very few data on the ecological requirements of most freshwater nematodes, it is difficult to determine the environmental quality of a biotope from its nematodes, let alone to predict the presence or abundance of a given nematode for a given habitat. Indeed, "Nematode diversity seems to lack any convincing consistent patterns in either particular environmental causes or ecological processes" (Hodda et al. 2009).

In the present study we analyzed the nematode communities in a large sample of springs in Trentino (Northern Italy). Given the expected difficulties in finding consistent patterns in nematode communities, we analyzed our data using several statistical approaches, with three major aims: first, of describing possible patterns of nematode distribution, second of assessing the ecological variables affecting community composition, and third of analyzing the associations between environmental conditions and nematode ecology. The present research contributes to our knowledge of the ecology of nematodes inhabiting the crenic habitat.

\section{METHODS}

Sampling and ecological analyses of 108 Alpine springs in the Autonomous Province of Trento (southeastern Alps, Italy), from June to November 2005, were organised by the Trentino Nature \& Science Museum. Nematodes were sampled by collecting sediment (sand, gravel) and submerged bryophyte or algae, according to a standardized protocol. In each spring two sediment areas were selected and sampled using a syringe. In addition, when present, about $13 \mathrm{~g}$ (w.w.) of submerged bryophyte were collected (see also Stoch et al. 2011, this issue, for further details). Nematode communities from 94 springs were examined.

Samples were fixed in formaldehyde ( $4 \%$ final concentration) and nematodes extracted using the silica gel method (Ludox TM 50, mesh size: $30 \mu \mathrm{m}$ ), modified from Pfannkuche \& Thiel (1988). Transfer and mounting of nematodes on permanent slides followed standard methods (Seinhorst 1959). Nematode community composition was investigated at two different spatial scales. Within springs, we investigated differences in populations between bryophytes and other substrata, while analyses of variation in nematode communities between springs, main focus of this study, was assessed by pooling all individuals from all substrata in a given spring.

Sample diversity was assessed using the Shannon and the Berger-Parker diversity indices (Magurran 2004), as well as taxonomic distinctness indices, measures of biological diversity that, unlike the other diversity indices, also take taxonomic distance into account by calculating averaged taxonomic distances among individuals in the community (Clarke \& Warwick 1998, $1999,2001)$. In particular, using data on species presence or absence, we calculated the Average Taxonomic Distinctness $\left(\Delta^{+}\right)$for each spring, reflecting the unevenness of the taxonomic tree, and its variation $\left(\Lambda^{+}\right)$proportional to the diversity due to the highest taxonomic levels (Warwick \& Clarke 1995). Using the established hierarchic taxonomy, taxonomic differences between species were calculated, coding differences between species of the same genus as 1 , the same family, order and class as 2, 3 and 4 respectively, and then scaling steps between taxonomic levels in proportion to the reduction in the number of categories (omitting this last stage did not change the results because the two taxonomic distance matrices were virtually identical, with $r$ $=0.999$; other details not shown). Observed $\Delta^{+}$and $\Lambda^{+}$ values were compared to the expected values using randomisation tests (with 999 permutations) based on the local master species list.

The $\beta$ diversity was calculated using the Whittaker formula $\beta=(S / \bar{a})-1$, where $S$ is the total number of species recorded in the study system, and $\bar{a}$ is the average number of species (alpha diversity) found in our springs [Whittaker 1972, see Wilson \& Shmida (1984) for a discussion on $\beta$-diversity measurements]. We selected the 46 springs with abundant specimens (i.e., 50 or more identified nematode specimens) and calculated the $\beta$ diversity values between pairs of springs of adjacent altitudes, from $210 \mathrm{~m}$ to $1970 \mathrm{~m}$, irrespective of the geographical position of springs. This is justified, as nematode species have intercontinental distribution, so that their presence in a given spring is related only to ecological conditions. In addition, $\beta$ diversity is always related to habitat gradients and not to single ecological parameters, justifying its analysis in relation to altitude only.

The Maturity Index (MI), an ecosystem parameter used in biomonitoring based on nematode community composition, was calculated according to Bongers (1990). Nematode families or genera can be given a score reflecting their position along a colonizer-persistent (c-p) gradient (partially reflecting the $r-K$ strategist gradient). MI is the weighted mean value of c-p scores (from 1 to 5) assigned to nematodes, and is proportional to the degree of naturalness and stability of a given biotope (Bongers 1990). Its values usually range from less than 2 , in very nutrient-enriched, disturbed systems, to about 4 in undisturbed, pristine environments (Bongers \& Ferris 1999). An alternative way to relate nematodes to their habitats is to group nematode species into guilds and to relate guilds rather than species to ecological parameters. For this purpose, we propose the guilds shown in table 1 , where freshwater nematodes are classified into seven guilds based both on their c-p values and on the type of food eaten by each taxon (Yeates et al. 1993). Nematode weight was calculated according to Andrássy (1956). 
Tab. 1. Proposed guilds for freshwater nematodes. Maturity c-p (colonizerspersisters) value. Principal food type: $\mathrm{b}=$ bacteria; $\mathrm{u}=$ unicellular eukaryotes; $\mathrm{p}=$ small animal predators; $\mathrm{o}=$ omnivorous with odontostyle. Nematode classification follows De Ley et al. (2006).

\begin{tabular}{|c|c|c|c|}
\hline Guild & $c-p$ & Food & Taxa \\
\hline A & 5 & $o, p$ & $\begin{array}{l}\text { Nygolaimina, Actinolaimidae, Discolaiminae, Belondiridae, } \\
\text { Thornenematidae, Aporcelaimidae }\end{array}$ \\
\hline B & 4 & o & Qudsianematidae, Nordiidae, Dorylaimidae, Tylencholaimoidea \\
\hline $\mathrm{C}$ & $3-4$ & $u, p$ & Mononchida, Tripylina, Tobriloidea, Ironina \\
\hline $\mathrm{D}$ & $2-3$ & $\mathrm{~b}, \mathrm{u}$ & Odontolaimidae, Chromadorida \\
\hline $\mathrm{E}$ & $2-3$ & $\mathrm{~b}$ & $\begin{array}{l}\text { Aphanolaimidae, Metateratocephalidae, Bastianiidae, } \\
\text { Teratocephalidae, Prismatolaimidae, Alaimidae, Monhysterida, } \\
\text { Plectoidea, Cylindrolaiminae, Desmodorida, Cephalobidae }\end{array}$ \\
\hline $\mathrm{F}$ & 1 & $\mathrm{~b}, \mathrm{u}, \mathrm{p}$ & Neodiplogastridae, Diplogaster, Butlerius \\
\hline G & 1 & $\mathrm{~b}$ & Rhabditomorpha, most Diplogastridae, Panagrolaimidae \\
\hline
\end{tabular}

Several independent variables that may influence the nematode community composition were recorded for each spring, namely altitude, water temperature, lithology (as a dichotomous variable, on carbonate or crystalline rocks), $\mathrm{pH}$, dissolved organic carbon (DOC), total amount of $\mathrm{N}, \mathrm{Ca}, \mathrm{Fe}$ in the waters, a four-level scale of flow velocity $(1=$ slow to 4 = very fast $)$, a fourlevel factor accounting for debris type $(\mathrm{R}=$ rocks, $\mathrm{S}=$ stones, $\mathrm{P}=$ pebbles, $\mathrm{M}=$ mud) and a dichotomous variable accounting for scarce or abundant vegetation. More details can be found in Cantonati et al. (2007).

Preliminary analyses on the environmental variables showed that water temperature was strongly and negatively correlated with altitude $(r=-0.81)$. We therefore disregarded the latter variable for the subsequent analyses. Other covariates and dichotomous variables did not raise collinearity issues (variance inflation factor $\leq 3.2$ ) and therefore could be entered simultaneously in the analyses. The variability in the diversity indices and in the MI with environmental variables was investigated by robust regressions, as the dependent variables were not Gaussian.

Eighty springs (38 on carbonate and 42 on crystalline rocks) were retained as significant for the multivariate analyses of community composition, while 14 were discarded because samples contained less than 8 specimens, a threshold under which we considered samples unrepresentative. The robustness of the results was checked with respect to this arbitrary threshold by rerunning the main statistical analyses including all springs. Community composition was first investigated by Principal Components Analyses on the covariance matrices of the whole data set and on different subsets, to facilitate interpretation of the results (see also below). This analysis was also run on guild, rather than species, composition of each community. Second, species community composition in relation to environmental variables was analyzed by distance-based redundancy analysis (db-RDA) (Legendre \& Anderson 1999), based on Bray-Curtis dissimilarities on untransformed abundances, with the ADONIS algorithm in the R package VEGAN 1.15-3 (Dixon 2003, Oksanen et al. 2009). This analysis, like MANOVA (Anderson 2001;
McArdle \& Anderson 2001) and AMOVA (Excoffier et al. 1992), partitions distance matrices among sources of variation and fits linear models to distance matrices. Predictor's significance is assessed by permutation tests with pseudo-F ratios. Final models were assessed with a forward variable selection procedure. In the first step predictors were entered one at time, their pseudo-F values and significances recorded, and the most significant predictor chosen. In the second step, all other independent variables were entered individually after the most significant predictor, and the most significant was chosen. The procedure stops when no significant variable is found. Model significance was assessed by an ANOVAlike permutation test for the joint effect of constraints in a Constrained Analysis of Principal Coordinates (CAP), an ordination method similar to redundancy analysis that allows non-Euclidean dissimilarity indices (Legendre \& Legendre 1998).

The generality of the results from db-RDA was checked by re-running the same analyses with the same variable selection procedure separately on different subsets of data. First, springs were randomly assigned to two subsamples (A: 43 springs with 82 nematode species; B: 37 springs with 72 species). Second, all analyses were re-run in subsets, first including only the 24 species for which at least 50 individuals were sampled (most abundant species, see also the Results), and then the species accounting for not less than $60 \%$ of individuals (11 species) and the springs accounting for not less than $50 \%$ of the sampled individuals (18 springs, minimal subset). To facilitate the interpretation of the multivariate statistical analysis results, abundances of each of the 24 most common species in the 80 springs (with more than 7 specimens) were related to each independent variable by Generalized Linear Models (GLM) for count data. Given that several models showed large overdispersion, often resulting from an excess of zeros, we used a Poisson distribution with overdispersion correction when the dispersion parameter was below 15 . More severely overdispersed models were analyzed using a negative binomial GLM or a Zero Inflated Poisson (ZIP) model according to their AICc values (Zuur 2009). Given the large number of statistical tests, the $\alpha$ - 
level of the latter univariate analyses was lowered according to the False Discovery Rate (FDR) procedure to control the proportion of type I errors (Verhoeven et al. 2005).

Nematodes were sampled from both sediments and mosses in each spring whenever present (see above). Since mosses create a very different micro-environment to sediments, they may host very different nematode communities. In order to investigate whether this occurs, we distinguished the nematode community found in mosses from that found on all other substrata from the same spring (in springs with mosses). These data were then analyzed by db-RDA of the whole dataset, as well as the 24 most abundant species. A dichotomous variable accounting for whether a sample was taken from mosses or other substrata was entered as a fixed factor, while the spring was entered as a random factor. Such an analysis can be performed by the ADONIS algorithm even if it does not explicitly allow inclusion of random effects, because, using ADONIS, the significance of a fixed factor does not change, irrespective of whether a second factor is fixed or random, as in an ANOVA-like partitioning of sum of squares (see Zar 1999 for the partitioning of sums of squares in ANOVA models and Legendre \& Legendre 1998 for details on db-RDA). Preference of each of the 24 most abundant nematode species for mosses or other substrata was investigated using Poisson Generalized Linear Mixed Models (GLMM) with an overdispersion correction, where the dichotomous variable accounting for a sample being taken from mosses or other substrata was entered as a fixed factor, and spring was entered as a random factor. These analyses were performed with the lmer procedure in the lme4 package (Bates et al. 2008).

The association between nematode ecology and environmental features of springs was investigated by the $4^{\text {th }}$-corner analysis (Legendre 1998; Dray \& Legendre 2008) applied to the sample of those 80 springs with more than 7 specimens. This analysis allowed statistical assessment of the link between the parameters in one matrix, describing ecological characters of species (A), and those in another describing environmental features of sampling sites (B), by multiplying these matrices, and the matrix representing the abundance of each species at each site $(\mathbf{C})$, namely $\mathbf{D}=\mathbf{B} \mathbf{C}^{\prime} \mathbf{A}$, where $\mathbf{D}$ is the matrix of the resulting association coefficients (Pearson correlation coefficients for two quantitative variables, Pearson $\chi^{2}$ for two qualitative variables and pseudo-F for one quantitative a one qualitative variable). Significance was assessed by permutation following Doledec et al. (1996) procedure with 999 permutations.

R 2.8.1 (R Development Core Team 2008) was used for all statistical analyses.

\section{RESULTS}

The 94 springs range in altitude from 170 to $2792 \mathrm{~m}$ a.s.l. (Tab. 2). 90 nematodes species were found, with a total of 5950 identified individuals (Tab. 3). On average each spring hosted 8.7 (5.7 SD, range 1-26) nematode species. The Chao (1987) incidence-based estimate of total number of nematode species in all springs in Trentino is 103 (8 SE) (other estimates gave similar results, details not shown).

The rank-abundance plot for the 90 nematode species is shown in figure 1. The group formed by the two, hardly distinguishable, species (especially in juveniles) Eumonhystera filiformis and E. vulgaris, was the most abundant with 649 individuals, followed by Dorylaimus stagnalis, Epitobrilus allophysis, Ethmolaimus pratensis and Monhystera paludicola with 573, 484, 392 and 309 individuals respectively. 24 species were represented by more than 60 individuals and accounted for more than $82 \%$ of the individuals. All nematode species are rather common in various freshwater environments, except for Eumonhystera tatrica, a very rare nematode described by Daday (1896) and never definitively found again. The measurements of our specimens were: $\mathrm{L}=0.32$ $0.35 \mathrm{~mm} ; \mathrm{a}=24 ; \mathrm{b}=4.5 ; \mathrm{c}=3.8 ; \mathrm{c}^{\prime}=7.5-9.5 ; \mathrm{V}=62 \%$ (where $\mathrm{L}=$ body length; $\mathrm{a}=\mathrm{L} /$ maximum body width; $\mathrm{b}$ $=\mathrm{L} /$ pharyngeal length; $\mathrm{c}=\mathrm{L} /$ tail length $; \mathrm{c}^{\prime}=$ tail length/anal body width; $\mathrm{V}=100 \times$ vulva distance from anterior end $/ \mathrm{L}$ ); cephalic diameter $=6 \mu \mathrm{m}$; body at cardia $=13 \mu \mathrm{m}$, head amphids at 1.7-1.8 head diameters from anterior end; body width/head width $=2.2$; vulvaanus/tail length $=0.41$. Some tylenchids and mermithids were also found in our samples, but we disregarded them as not being typical free-living nematodes.

For the total set of springs, Shannon diversity index (base e) was 3.53 and $\Delta^{+}$was 88.5 . Figure 2 shows $\Delta^{+}$ and $\Lambda^{+}$values and their confidence limits. One spring $\left(H^{\prime}=\mathrm{C} 0962 \mathrm{~V}\right.$, see table 2 for abbreviations $)$ has higher, while two $\left(D^{\prime}=\right.$ BR0658 and $\left.i=\mathrm{CV} 1084\right)$ have lower than expected taxonomic diversity for their number of species. Four springs, all situated $>1000 \mathrm{~m}$ a.s.l., $(i=$ CV1084, $t=\mathrm{AD} 1790, u^{\prime}=\mathrm{LT} 1911$ and $y^{\prime}=$ OC2792), have larger than expected $\Lambda^{+}$values. Spring $i$ has lower than expected $\Delta^{+}$and higher $\Lambda^{+}$values (Fig. 2). This is due to the fact that the five species found in that spring pertain to only two superior (order level) taxa (Monhysterida and Dorylaimida). Therefore this community has low taxonomic diversity but large taxonomic variation, i.e. diversity of spring $i$ is almost exclusively based, not on genus or family level diversity (since 3 species pertain to the same genus Eumonhystera), but on higher taxon (i.e. order) diversity.

The overall $\beta$ diversity for our 94 springs was 9.4, indicating a very low average replacement rate of nematode species along environmental gradients. The mean $\beta$ diversity values calculated on pairs of springs of adjacent altitude (from the 46 springs with abundant specimens, see Methods), were related to the mean altitude of the pairs of springs. The changing rates of compositional turnover along the altitudinal gradient shows four peaks, at about $650 \mathrm{~m}, 1200 \mathrm{~m}, 1450 \mathrm{~m}, 1700-1850$ and $1950 \mathrm{~m}$ a.s.l., which indicate transitions between nematode community types at those altitudes (other details not shown). 
Tab. 2. List of springs with more than 7 nematode specimens, ranked by altitude, from lowest $(170 \mathrm{~m})$ to highest $(2792 \mathrm{~m})$. The second part of the code refers to the altitude (m a.s.1.); abbreviations refer to the letters used in figures; number of individuals; number of species collected at each spring; percentage of the most abundant species (Berger-Parker index) at each spring where number of individuals $\geq 50$.

\begin{tabular}{|c|c|c|c|c|c|c|c|c|c|}
\hline Springs & abbrev. & indiv. & spp. & B-P & Springs & abbrev. & indiv. & spp. & B-P \\
\hline $\mathrm{BC} 0170$ & A & 82 & 17 & 30 & BR1315 & $\mathrm{N}$ & 130 & 17 & 73 \\
\hline SC0250 & $\mathrm{a}$ & 67 & 16 & 22 & CV1350 & $\mathrm{N}$ & 14 & 4 & - \\
\hline BS0420 & $a^{\prime}$ & 38 & 6 & - & AD1353 & $\mathrm{n}^{\prime}$ & 22 & 3 & - \\
\hline LD0420 & B & 100 & 16 & 27 & BR1358 & $\mathrm{O}$ & 54 & 5 & 70 \\
\hline AN0430 & $\mathrm{B}^{\prime}$ & 61 & 13 & 51 & LD1400 & $\mathrm{O}^{\prime}$ & 61 & 13 & 30 \\
\hline PG0453 & $\mathrm{b}$ & 10 & 3 & - & CV1421 & $\mathrm{o}^{\prime}$ & 52 & 12 & 25 \\
\hline $\mathrm{BC} 0503$ & $\mathrm{C}$ & 37 & 5 & - & CV1435 & $\mathrm{P}$ & 78 & 5 & 45 \\
\hline LD0509 & $\mathrm{C}^{\prime}$ & 14 & 4 & - & BR1436 & $\mathrm{P}^{\prime}$ & 67 & 2 & 72 \\
\hline LD0584 & $c^{\prime}$ & 94 & 10 & 41 & AN1474 & $\mathrm{P}$ & 50 & 10 & 18 \\
\hline AN0590 & $\mathrm{D}$ & 192 & 18 & 31 & BS1527 & Q & 32 & 3 & - \\
\hline BR0658 & $\mathrm{D}^{\prime}$ & 56 & 7 & 77 & MP1566 & $\mathrm{Q}^{\prime}$ & 18 & 8 & - \\
\hline BR0679 & $\mathrm{d}$ & 89 & 11 & 40 & CV1575 & $\mathrm{q}$ & 7 & 3 & - \\
\hline BS0705 & $\mathrm{d}^{\prime}$ & 32 & 12 & - & BR1605 & $\mathrm{R}$ & 68 & 9 & 54 \\
\hline LD0720 & $\mathrm{E}$ & 157 & 16 & 35 & CV1623 & $\mathrm{R}^{\prime}$ & 145 & 16 & 26 \\
\hline BR0735 & $E^{\prime}$ & 29 & 15 & - & CA1642 & $\mathrm{r}$ & 23 & 10 & - \\
\hline VF0745 & $\mathrm{e}$ & 10 & 4 & - & AD1654 & $r^{\prime}$ & 34 & 6 & - \\
\hline AT0756 & $\mathrm{e}^{\prime}$ & 85 & 19 & 18 & CV1655 & $\mathrm{S}$ & 38 & 7 & - \\
\hline BR0790 & $\mathrm{F}^{\prime}$ & 173 & 17 & 23 & AD1665 & $S^{\prime}$ & 47 & 13 & - \\
\hline BR0804 & $\mathrm{f}$ & 43 & 13 & - & MD1670 & $\mathrm{s}$ & 164 & 16 & 34 \\
\hline CV0854 & $\mathrm{f}^{\prime}$ & 17 & 5 & - & AN1685 & $\mathrm{s}^{\prime}$ & 75 & 9 & 44 \\
\hline AD0905 & $\mathrm{G}$ & 147 & 26 & 24 & SL1724 & $\mathrm{T}^{\prime}$ & 76 & 8 & 34 \\
\hline LD0928 & $\mathrm{G}^{\prime}$ & 75 & 9 & 48 & AD1790 & $\mathrm{t}$ & 57 & 9 & 68 \\
\hline LD0930 & $\mathrm{g}$ & 10 & 6 & - & MD1841 & $t^{\prime}$ & 93 & 10 & 45 \\
\hline BR0950 & $\mathrm{g}^{\prime}$ & 34 & 3 & - & AD1853 & $\mathrm{U}$ & 182 & 20 & 58 \\
\hline CV0962 & $\mathrm{H}$ & 47 & 6 & - & CV1855 & $U^{\prime}$ & 39 & 6 & - \\
\hline AT0972 & $\mathrm{H}^{\prime}$ & 24 & 8 & - & PS1880 & $\mathrm{u}$ & 24 & 5 & - \\
\hline OC0981 & $\mathrm{h}$ & 143 & 8 & 52 & LT1911 & $\mathrm{u}^{\prime}$ & 250 & 17 & 17 \\
\hline AN1000 & $h^{\prime}$ & 26 & 5 & - & CV1940 & $\mathrm{V}$ & 194 & 16 & 31 \\
\hline AT1052 & I & 230 & 8 & 68 & AD1944 & $V^{\prime}$ & 123 & 16 & 33 \\
\hline AD1077 & $I^{\prime}$ & 44 & 7 & - & AN1950 & $\mathrm{v}$ & 64 & 5 & 61 \\
\hline CV1084 & $\mathrm{i}$ & 41 & 5 & - & AD1990 & $\mathrm{v}^{\prime}$ & 117 & 7 & 49 \\
\hline MC1115 & $\mathrm{i}^{\prime}$ & 112 & 9 & 45 & CV2085 & $\mathrm{w}$ & 15 & 5 & - \\
\hline AD 1125 & $\mathrm{~K}$ & 53 & 13 & 26 & CV2126 & $\mathrm{w}^{\prime}$ & 56 & 14 & 23 \\
\hline VZ1178 & $\mathrm{k}$ & 62 & 12 & 18 & AD2153 & $\mathrm{X}$ & 14 & 4 & - \\
\hline CV1200 & $\mathrm{k}^{\prime}$ & 20 & 7 & - & CV2182 & $\mathrm{x}$ & 102 & 16 & 32 \\
\hline CV1215 & $\mathrm{L}$ & 187 & 11 & 41 & BR2240 & $\mathrm{x}^{\prime}$ & 28 & 7 & - \\
\hline AD1235 & $L^{\prime}$ & 75 & 6 & 88 & OC 2278 & Y & 193 & 18 & 37 \\
\hline PS1250 & $l^{\prime}$ & 16 & 4 & - & AD2314 & $\mathrm{Y}^{\prime}$ & 16 & 6 & - \\
\hline CV1254 & M & 83 & 11 & 42 & AD2739 & $\mathrm{y}$ & 25 & 3 & - \\
\hline AD 1300 & $\mathrm{~m}^{\prime}$ & 155 & 22 & 25 & OC2792 & $y^{\prime}$ & 78 & 7 & 36 \\
\hline
\end{tabular}

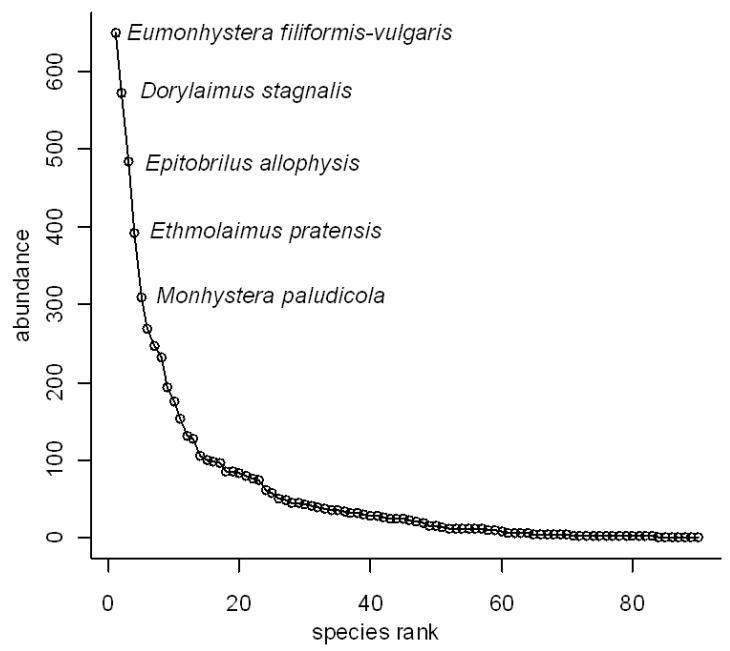

Fig. 1. Rank-abundance-plot for the 90 nematode species in the entire sample of 94 springs. 
Tab. 3. List of nematode species from the CRENODAT project. Columns: numerical codes for species used in figures, names of taxa, c-p values, trophic group, average adult weight ( $\mu$ g wet weight), guild (see Tab.3), number of springs in which a species was found, total number of individuals found in the 80 springs with more than 7 specimens.

\begin{tabular}{|c|c|c|c|c|c|c|c|c|}
\hline & Species & Taxon of Tab.1 & $\mathrm{c}-\mathrm{p}$ & food & $\mu \mathrm{g}$ & guild & \# springs & \# indiv. \\
\hline 1 & Achromadora terricola (De Man, 1880) & Chromadorida & 3 & unicell euk & 0.61 & $\mathrm{D}$ & 19 & 132 \\
\hline 2 & Alaimus primitivus (De Man, 1880) & Alaimidae & 4 & bact & 0.19 & $\mathrm{E}$ & 10 & 39 \\
\hline 3 & Amphidelus sp.1 & Alaimidae & 4 & bact & 0.35 & $\mathrm{E}$ & 3 & 15 \\
\hline 4 & Anaplectus granulosus (Bastian, 1865) & Plectoidea & 2 & bact & 0.92 & $\mathrm{E}$ & 2 & 2 \\
\hline 5 & Anatonchus tridentatus (De Man, 1876) & Mononchida & 4 & pred & 7.74 & $\mathrm{C}$ & 8 & 38 \\
\hline 6 & Aphanolaimus aquaticus Daday, 1894 & Aphanolaimidae & 3 & pred & 0.66 & $\mathrm{E}$ & 14 & 58 \\
\hline 7 & Aporcelaimellus krygeri (Ditlevsen, 1928) & Aporcelaimidae & 5 & pred & 22.14 & A & 11 & 36 \\
\hline 8 & Aporcelaimellus obtusicaudatus (Bastian, 1865) & Aporcelaimidae & 5 & pred & 16.25 & A & 8 & 44 \\
\hline 9 & Aporcelaimellus sp.1 & Aporcelaimidae & 5 & pred & 9.45 & A & 1 & 1 \\
\hline 10 & Bastiania gracilis De Man, 1876 & Bastianiidae & 3 & bact & 0.12 & $\mathrm{E}$ & 3 & 20 \\
\hline 11 & Cephalobus persegnis Bastian, 1865 & Cephalobidae & 2 & bact & 0.37 & $\mathrm{E}$ & 1 & 1 \\
\hline 12 & Ceratoplectus armatus (Bütschli, 1873) & Plectoidea & 2 & bact & 0.12 & $\mathrm{E}$ & 2 & 2 \\
\hline 13 & Clarkus papillatus Bastian, 1865 & Mononchida & 4 & pred & 1.51 & $\mathrm{C}$ & 9 & 25 \\
\hline 14 & Crocodorylaimus sp.1 & Dorylaimidae & 4 & omniv & 1.56 & $\mathrm{~B}$ & 7 & 33 \\
\hline 15 & Cylindrolaimus bambus Andrássy, 1968 & Cylindrolaiminae & 3 & bact & 0.13 & $\mathrm{E}$ & 4 & 11 \\
\hline 16 & Cylindrolaimus obtusus Cobb, 1916 & Cylindrolaiminae & 3 & bact & 0.16 & $\mathrm{E}$ & 4 & 10 \\
\hline 17 & Dorylaimus stagnalis Dujardin, 1845 & Dorylaimidae & 4 & omniv & 29.72 & $\mathrm{~B}$ & 37 & 560 \\
\hline 18 & Enchodelus sp.1 & Nordiidae & 4 & omniv & 2.59 & $\mathrm{~B}$ & 13 & 49 \\
\hline 19 & Enchodelus vesuvianus (Cobb, 1893) & Nordiidae & 4 & omniv & 1.86 & $\mathrm{~B}$ & 3 & 3 \\
\hline 20 & Epidorylaimus agilis (De Man, 1880) & Qudsianematidae & 4 & omniv & 2.43 & $\mathrm{~B}$ & 8 & 13 \\
\hline 21 & Epidorylaimus consobrinus (De Man, 1918) & Qudsianematidae & 4 & omniv & 1.82 & $\mathrm{~B}$ & 1 & 3 \\
\hline 22 & Epidorylaimus pseudoagilis (Altherr, 1952) & Qudsianematidae & 4 & omniv & 1.82 & $\mathrm{~B}$ & 34 & 125 \\
\hline 23 & Epitobrilus allophysis (Steiner, 1919) & Tobriloidea & 4 & small euk & 3.66 & $\mathrm{C}$ & 44 & 483 \\
\hline 24 & Ethmolaimus pratensis De Man, 1880 & Chromadorida & 3 & bact & 0.26 & $\mathrm{D}$ & 34 & 392 \\
\hline 25 & Eucephalobus oxyuroides (De Man, 1876) & Cephalobidae & 2 & bact & 0.25 & $\mathrm{E}$ & 1 & 2 \\
\hline 26 & Eudorylaimus sp.1 & Qudsianematidae & 4 & omniv & 2.84 & $\mathrm{~B}$ & 33 & 86 \\
\hline 27 & Eudorylaimus sp.2 & Qudsianematidae & 4 & omniv & 2.24 & $\mathrm{~B}$ & 3 & 6 \\
\hline 28 & Eumonhystera barbata Andrássy, 1981 & Monhysterida & 2 & bact & 0.14 & $\mathrm{E}$ & 26 & 232 \\
\hline 29 & Eumonhystera dispar (Bastian, 1865) & Monhysterida & 2 & bact & 0.28 & $\mathrm{E}$ & 10 & 41 \\
\hline 30 & Eumonhystera filiformis-vulgaris (Bastian, 1865-De Man, 1880) & Monhysterida & 2 & bact & 0.25 & $\mathrm{E}$ & 43 & 649 \\
\hline 31 & Eumonhystera hungarica Andrássy, 1981 & Monhysterida & 2 & bact & 0.02 & $\mathrm{E}$ & 2 & 21 \\
\hline 32 & Eumonhystera longicaudatula (Gerlach\&Riemann, 1973) & Monhysterida & 2 & bact & 0.07 & $\mathrm{E}$ & 3 & 4 \\
\hline 33 & Eumonhystera similis (Bütschli, 1873) & Monhysterida & 2 & bact & 0.13 & $\mathrm{E}$ & 4 & 84 \\
\hline 34 & Eumonhystera sp.1 & Monhysterida & 2 & bact & 0.15 & $\mathrm{E}$ & 3 & 3 \\
\hline 35 & Eumonhystera tatrica (Daday, 1896) & Monhysterida & 2 & bact & 0.07 & $\mathrm{E}$ & 4 & 11 \\
\hline 36 & Fictor fictor (Bastian, 1865) & Neodiplogastridae & 3 & small euk & 0.84 & $\mathrm{~F}$ & 5 & 32 \\
\hline 37 & Geomonhystera steineri (Micoletzky, 1922) & Monhysterida & 2 & bact & 0.28 & $\mathrm{E}$ & 4 & 5 \\
\hline 38 & Hofmaenneria brachystoma (Hofmänner, 1914) & Monhysterida & 3 & bact & 0.15 & $\mathrm{E}$ & 3 & 6 \\
\hline 39 & Ironus longicaudatus De Man, 1884 & Ironina & 4 & small euk & 1.25 & $\mathrm{C}$ & 3 & 27 \\
\hline 40 & Ironus paramacramphis Altherr, 1972 & Ironina & 4 & small euk & 15.94 & $\mathrm{C}$ & 15 & 78 \\
\hline 41 & Ironus tenuicaudatus De Man, 1876 & Ironina & 4 & small euk & 10.58 & $\mathrm{C}$ & 5 & 43 \\
\hline 42 & Laimydorus sp.1 & Dorylaimidae & 4 & omniv & 7.23 & $\mathrm{~B}$ & 1 & 1 \\
\hline 43 & Mesodorylaimus bastiani (Bütschli, 1873) & Dorylaimidae & 4 & omniv & 2.12 & $\mathrm{~B}$ & 2 & 3 \\
\hline 44 & Mesodorylaimus centrocercus (De Man, 1880) & Dorylaimidae & 4 & omniv & 1.91 & $\mathrm{~B}$ & 2 & 2 \\
\hline 45 & Mesodorylaimus sp.1 & Dorylaimidae & 4 & omniv & 2.01 & $\mathrm{~B}$ & 4 & 8 \\
\hline 46 & Metateratocephalus crassidens (De Man, 1880) & Metateratocephalidae & 3 & bact & 0.08 & $\mathrm{E}$ & 3 & 3 \\
\hline 47 & Metateratocephalus gracilicaudatus Andrássy, 1985 & Metateratocephalidae & 3 & bact & 0.04 & $\mathrm{E}$ & 3 & 7 \\
\hline 48 & Monhystera paludicola De Man, 1881 & Monhysterida & 2 & bact & 0.75 & $\mathrm{E}$ & 16 & 308 \\
\hline 49 & Monhystrella paramacrura (Meyl, 1953) & Monhysterida & 2 & bact & 0.04 & $\mathrm{E}$ & 1 & 1 \\
\hline 50 & Mononchus aquaticus Coetzee, 1968 & Mononchida & 4 & pred & 2.69 & $\mathrm{C}$ & 2 & 8 \\
\hline 51 & Mononchus pulcher Andrássy, 1993 & Mononchida & 4 & pred & 3.13 & $\mathrm{C}$ & 6 & 34 \\
\hline 52 & Mononchus truncatus Bastian, 1865 & Mononchida & 4 & pred & 4.59 & $\mathrm{C}$ & 25 & 154 \\
\hline 53 & Mylonchulus sigmaturus Cobb, 1917 & Mononchida & 4 & pred & 1.27 & $\mathrm{C}$ & 1 & 3 \\
\hline 54 & Odontolaimus aquaticus Schneider, 1937 & Odontolaimidae & 3 & small euk & 0.13 & $\mathrm{D}$ & 5 & 12 \\
\hline 55 & Oxydirus oxycephaloides (De Man, 1921) & Belondiridae & 5 & omniv & 2.81 & A & 1 & 11 \\
\hline 56 & Panagrellus pycnus Thorne, 1938 & Panagrolaimidae & 1 & bact & 3.72 & G & 2 & 29 \\
\hline 57 & Panagrellus redivivus (L., 1767) & Panagrolaimidae & 1 & bact & 4.54 & G & 9 & 45 \\
\hline 58 & Panagrolaimus rigidus (Schneider, 1866) & Panagrolaimidae & 1 & bact & 0.79 & G & 2 & 16 \\
\hline 59 & Paractinolaimus macrolaimus (De Man, 1880) & Actinolaimidae & 5 & pred & 9.19 & A & 16 & 193 \\
\hline 60 & Paramphidelus paramonovi (Eliashvili, 1971) & Alaimidae & 4 & bact & 0.1 & $\mathrm{E}$ & 2 & 4 \\
\hline 61 & Paravulvus hartingii (De Man, 1880) & Nygolaimina & 5 & pred & 1.54 & A & 2 & 6 \\
\hline 62 & Paraxonchium gr. laetificans & Qudsianematidae & 1 & bact & 10.33 & A & 1 & 2 \\
\hline 63 & Plectus acuminatus Bastian, 1865 & Plectoidea & 2 & bact & 1.12 & $\mathrm{E}$ & 28 & 97 \\
\hline
\end{tabular}


Tab. 3. Continuation.

\begin{tabular}{|c|c|c|c|c|c|c|c|c|}
\hline & Species & Taxon of Tab.1 & $c-p$ & food & $\mu \mathrm{g}$ & guild & \# springs & \# indiv. \\
\hline 64 & Plectus andrassyi Timm, 1971 & Plectoidea & 2 & bact & 0.94 & $\mathrm{E}$ & 17 & 100 \\
\hline 65 & Plectus aquatilis Andrássy, 1985 & Plectoidea & 2 & bact & 1.72 & $\mathrm{E}$ & 6 & 35 \\
\hline 66 & Plectus decens Andrássy, 1985 & Plectoidea & 2 & bact & 0.32 & E & 1 & 1 \\
\hline 67 & Plectus exinocaudatus Truskova, 1976 & Plectoidea & 2 & bact & 0.05 & $\mathrm{E}$ & 1 & 4 \\
\hline 68 & Plectus longicaudatus Bủtschli, 1873 & Plectoidea & 2 & bact & 0.15 & E & 1 & 2 \\
\hline 69 & Plectus parietinus Bastian, 1865 & Plectoidea & 2 & bact & 2.12 & E & 30 & 244 \\
\hline 70 & Plectus parvus Bastian, 1865 & Plectoidea & 2 & bact & 0.12 & E & 5 & 21 \\
\hline 71 & Prionchulus muscorum (Dujardin, 1845) & Mononchida & 4 & pred & 8.49 & $\mathrm{C}$ & 15 & 85 \\
\hline 72 & Prionchulus punctatus Cobb, 1917 & Mononchida & 4 & pred & 6.86 & $\mathrm{C}$ & 3 & 5 \\
\hline 73 & Prismatolaimus dolichurus De Man, 1880 & Prismatolaimidae & 3 & bact & 0.26 & $\mathrm{E}$ & 7 & 75 \\
\hline 74 & Prismatolaimus intermedius (Bűtschli, 1873) & Prismatolaimidae & 3 & bact & 0.1 & $\mathrm{E}$ & 16 & 96 \\
\hline 75 & Prodesmodora zullinii Ocaňa, Abolafia \& Abebe, 2001 & Desmodorida & 3 & bact & 0.04 & $\mathrm{E}$ & 7 & 27 \\
\hline 76 & Prodorylaimus longicaudatoides Altherr, 1968 & Dorylaimidae & 4 & omniv & 14.48 & 4 & 3 & 11 \\
\hline 77 & Protorhabditis filiformis (Bủtschli, 1873) & Rhabditomorpha & 1 & bact & 0.1 & 1 & 1 & 1 \\
\hline 78 & Rhyssocolpus microdorus (Schiemer, 1965) & Nordiidae & 4 & omniv & 0.97 & B & 3 & 9 \\
\hline 79 & Semitobrilus cislongicaudatus (Gagarin, 1971) & Tobriloidea & 3 & small euk & 4.66 & $\mathrm{C}$ & 3 & 48 \\
\hline 80 & Semitobrilus pellucidus (Bastian, 1865) & Tobriloidea & 3 & small euk & 5.65 & $\mathrm{C}$ & 1 & 22 \\
\hline 81 & Teratocephalus terrestris (Bütschli, 1873) & Teratocephalidae & 3 & bact & 0.07 & $\mathrm{E}$ & 2 & 3 \\
\hline 82 & Theristus agilis (De Man, 1880) & Monhysterida & 2 & bact & 1.25 & $\mathrm{E}$ & 7 & 30 \\
\hline 83 & Tobrilus gracilis (Bastian, 1865) & Tobriloidea & 3 & small euk & 3.42 & $\mathrm{C}$ & 14 & 98 \\
\hline 84 & Tobrilus helveticus (Hofmänner, 1914) & Tobriloidea & 3 & small euk & 2.9 & $\mathrm{C}$ & 2 & 45 \\
\hline 85 & Tobrilus zakopanensis (Stefanski, 1924) & Tobriloidea & 3 & small euk & 3.99 & $\mathrm{C}$ & 1 & 5 \\
\hline 86 & Tripyla filicaudata De Man, 1880 & Tripylina & 3 & pred & 1.39 & $\mathrm{C}$ & 22 & 270 \\
\hline 87 & Tripyla glomerans Bastian, 1865 & Tripylina & 3 & pred & 10.16 & $\mathrm{C}$ & 4 & 25 \\
\hline 88 & Tripyla setifera Bütschli, 1873 & Tripylina & 3 & pred & 1.67 & $\mathrm{C}$ & 1 & 1 \\
\hline 89 & Trischistoma monohystera (De Man, 1880) & Tripylina & 3 & pred & 1.01 & $\mathrm{C}$ & 7 & 77 \\
\hline 90 & Tylencholaimus minimus (De Man, 1876) & Tylencholaimoidea & 4 & fungi & 0.22 & B & 19 & 176 \\
\hline
\end{tabular}
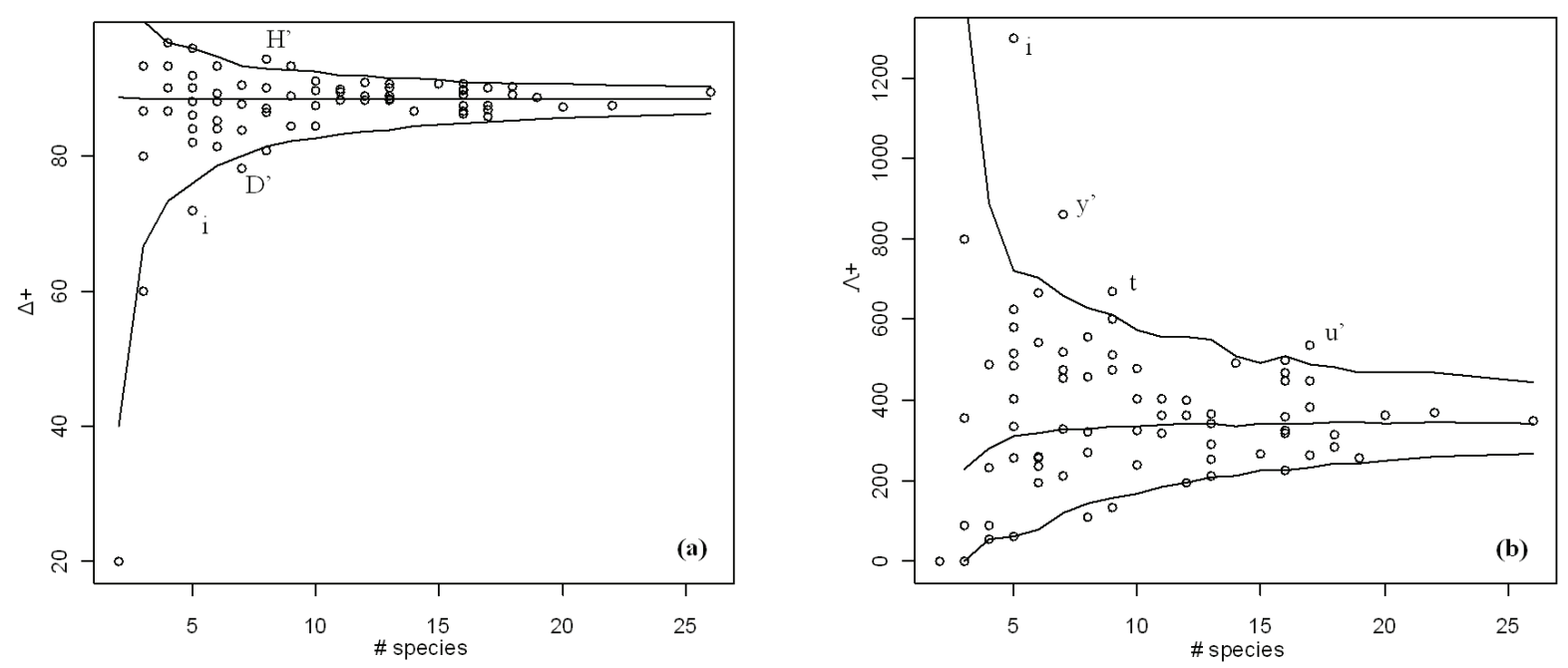

Fig. 2. (a) $\Delta+$ and (b) $\Lambda+$ indices for the 80 springs with more than 7 specimens in relation to the number of species in each sample. Lines represent empirical expected values and asymptotic $95 \%$ confidence interval.

Nematode community composition was first investigated by PCA on the minimal data subset (i.e. the 18 richest springs with the 11 most numerous nematode species). With reference to figure $3 \mathrm{a}$ we see that one set of species (Epitobrilus allophysis, Ethmolaimus pratensis, Eumonhystera barbata, Mononchus truncatus, Paractinolaimus macrolaimus, Plectus parietinus, Tripyla glomerans, Tylencholaimus minimus) prefers the same ecological conditions, while three (Dorylaimus stagnalis, Eumonhystera filiformis-vulgaris, Monhystera paludicola) are very (ecologically) different, both among each other and from the former set. When analyzing springs in the same subset we noted that nematode community composition of springs $h=$ OC0981, $I$ $=\mathrm{AT} 1052, L=\mathrm{CV} 1215, U=\mathrm{AD} 1853, V^{\prime}=\mathrm{CV} 1940, v^{\prime}$ $=$ AD1990, differs from that of most of the springs (Fig. $3 b)$. Similar results were obtained when we re-ran the analyses based on guild rather than species abundance (Fig. 3c-d). In particular, guild $\mathrm{E}$ is well separated from the others, as indicated by the large variance explained by the first principal component, which may be related to trophic distance between this and the other guilds. 

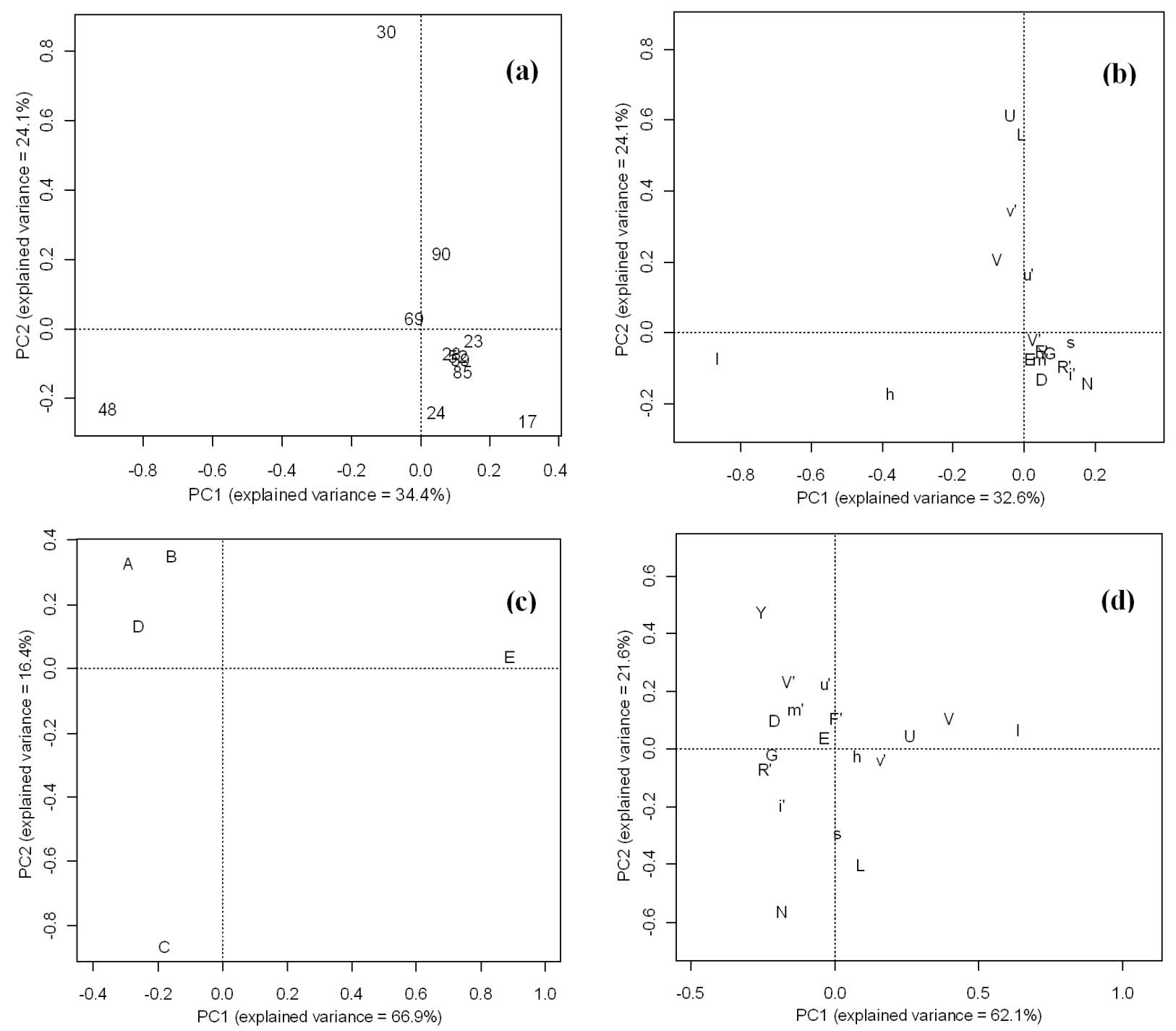

Fig. 3. Ordination plots from PCA on (a) the 11 most abundant species; (b) the 18 nematode-richest springs; (c) guilds; (d) the 18 nematode-richest springs, based on guild rather than species composition.

Springs $I=\mathrm{AT} 1052, L=\mathrm{CV} 1215, N=\mathrm{BR} 1315, V=$ CV1940, $Y=$ OC2278, seem to differ from the others in the autoecology of the nematode community.

The db-RDA analyses indicated that water temperature was the most important environmental variable shaping nematode community composition (Tab. 4). Indeed this variable was significant in all models. Lithology was significant in all models except for that on the minimal subset. Iron concentration was significant in the model based on the whole sample and in that from subsample $\mathrm{A}$, while $\mathrm{pH}$ was significant in the models based on subsample B (Tab. 4). Thus, water temperature and lithology seem to be the major determinants of nematode community composition, while no firm conclusion can be drawn for $\mathrm{pH}$ and iron concentration. The following analyses of nematode community composition against environmental variables were therefore based primarily on the effect of water temperature and lithology.
The results of the GLM analyses relating abundance of each of the 24 most abundant species to abiotic factors are summarized in table 5 . The main aim of this table is to provide information about the general abiotic determinants of the distribution of these species in springs, for which very poor, if any, information has been published. Given the mainly descriptive purpose of this table, the results will not be discussed in detail, except with respect to temperature and lithology, which seem to shape nematode community composition.

GLM analyses showed that the abundance of eight (of 24 most abundant) species varied significantly with water temperature (Tab. 5). Shannon, $\Delta^{+}$and $\Lambda^{+}$indices and total number of individuals sampled at each site did not vary significantly with water temperature (details not shown). Conversely, MI increased with water temperature (coef $0.08 \pm 0.03 \mathrm{SE}, \mathrm{t}_{77}=3.28, P<0.01$ ). The same results were obtained when we re-ran the analyses for all 94 springs (details not shown). 
Tab. 4. Final db-RDA models run on the different sets of data.

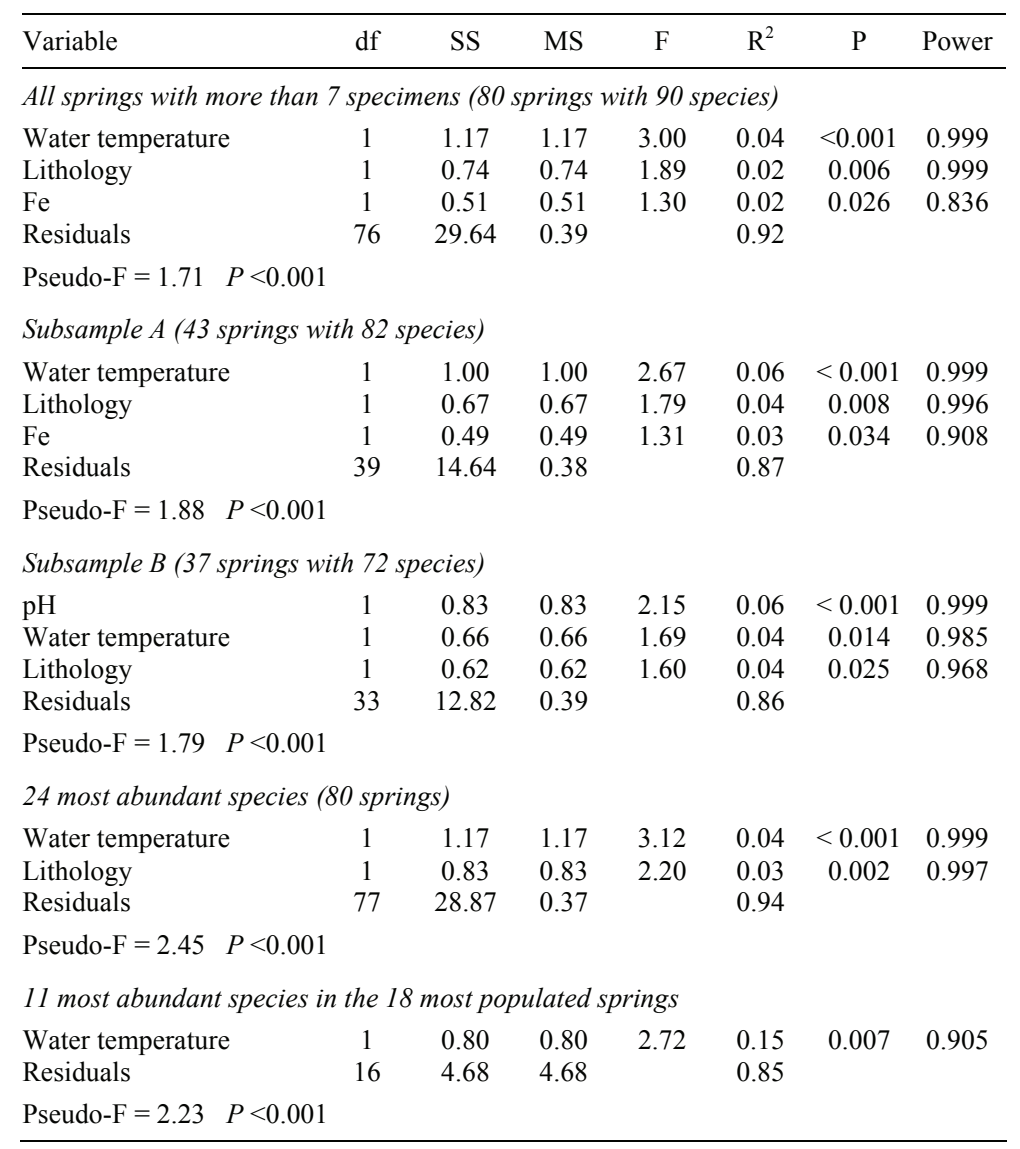

Based on the entire sample of 94 springs, a total of 77 and 78 nematode species were found on carbonate and crystalline rocks respectively. On average a spring on carbonate rock hosted $7.95 \pm 5.09$ SD nematode species, on crystalline soil $8.78 \pm 5.84$ SD species, with no significant difference in the mean number of species hosted by springs on either substratum $\left(\mathrm{t}_{92}=-0.73, P=\right.$ 0.47). Total number of species estimated with Chao's method was $91 \pm 8 \mathrm{SE}$ and $107 \pm 15 \mathrm{SE}$ for carbonate and crystalline substrata respectively. There is no evidence that differences in species composition arose from segregation of species on either substratum (details not shown). Conversely, since lithology also showed a significant influence in the analysis of the subset of the 24 most abundant species, its effect could be caused by differences in the abundance of individuals of common species on the substratum. Indeed, univariate analyses indicated that abundance of 8 of the 24 most common species differed significantly between lithologies (Tab. $5)$. However, no diversity index varied significantly with lithology ( $P \geq 0.16$, other details not shown).

Nematode communities in bryophytes differed significantly from those on other substrata, as shown by $\mathrm{db}$ RDAs based on both the full sample of species in 80 springs with more than 7 specimens (pseudo- $\mathrm{F}=3.94$, $\left.R^{2}=0.04, P<0.01\right)$ and the subset of the 24 most abundant species in the same springs (pseudo-F $=4.77, R^{2}=$
$0.05, P<0.01)$. GLMM analyses showed that Eumonhystera barbata and Paractinolaimus macrolaimus were more abundant in mosses than on the other substrata $(E$. barbata: $\mathrm{t}_{76}=-17.39, P<0.01 ; P$. macrolaimus: $\mathrm{t}_{76}=$ $-46.35, P<0.01$ ), abundance of the E. filiformis - E. vulgaris complex did not differ between substrata $\left(\mathrm{t}_{76}=\right.$ $-0.25, P=0.80$ ), while number of individuals of all other species was higher on other substrata than in mosses ( $\mathrm{t}_{76} \geq 3.14, P<0.01$ in all cases).

The $4^{\text {th }}$-corner analysis revealed associations between nematode ecology and environmental features of springs. In particular, c-p value was negatively correlated with altitude $(r=-0.15, P=0.02)$ but positively with water temperature $(r=0.22, P<0.01)$. Mean weigh of nematode species increased with $\mathrm{pH}(r=0.22, P$ $<0.01)$ and decreased with DOC $(r=-0.17, P<0.01)$. Abundance of nematode species feeding on different food types changed significantly with water temperature (pseudo-F $=109.16, P=0.01$ ). In particular, abundance of bacterial feeders decreases significantly with water temperature $(r=-0.16, P=0.01)$ while that of predators increased $(r=0.14, P<0.01)$. Abundance of omnivores and eukaryotic feeders was not affected by water temperature $(P \geq 0.29)$. Not surprising, guild abundance was also affected by water temperature, (pseudo-F $=99.4, P$ $=0.01$ ), with guilds $\mathrm{A}$ and $\mathrm{D}$ more abundant in warm (A: $r=0.19, P=0.01 ; \mathrm{D}: r=0.11, P=0.04$ ), and guild 
Tab. 5. Summary of the univariate GLMs relating abundance of each species to each environmental variable. + and $-:$ positive and negative regression coefficients; ++ and - - significantly positive and negative regressions after FDR correction; For categorical variables, the factor level where the species is most abundant is shown. Cr: crystalline rocks; Ca: carbonates; R: rocks; P: pebbles; M: mud; S: scarce vegetation; A: abundant vegetation; Water speed is expressed as 4 levels, indicating increasing water speed from 1 (still water) to 4 (fast running water); ${ }_{\mathrm{b}}$ : negative binomial GLM; $\mathrm{z}$ : zero-inflated Poisson (ZIP) GLM. All other models: Poisson GLM with overdispersion correction. All models are based on the sample of 80 springs with more than 7 nematode specimens.

\begin{tabular}{|c|c|c|c|c|c|c|c|c|c|c|c|}
\hline Species & Altitude & ithology & $\mathrm{pH}$ & DOC & $\mathrm{N}_{\text {tot }}$ & $\mathrm{Ca}$ & $\mathrm{Fe}$ & W. temp & W. speed & Debris type & Veget. \\
\hline Eumonhystera filiformis-vulgaris & $+_{b}$ & $\mathrm{Cr}_{\mathrm{b}}$ & $-{ }_{b}$ & $\boldsymbol{+}_{\mathrm{b}}$ & $-{ }_{b}$ & $-{ }_{b}$ & $-{ }_{b}$ & $--_{b}$ & $\mathbf{4}_{\mathrm{b}}$ & $\mathbf{R}_{\mathrm{b}}$ & $\mathbf{S}_{\mathrm{b}}$ \\
\hline Dorylaimus stagnalis & $-{ }_{b}$ & $\mathbf{C a} \mathbf{a}_{b}$ & $++_{b}$ & $++{ }_{b}$ & $-{ }_{b}$ & $+_{b}$ & $-{ }_{b}$ & $+_{b}$ & $2{ }_{b}$ & $\mathbf{P}_{\mathrm{b}}$ & $\mathbf{A}_{\mathrm{b}}$ \\
\hline Epitobrilus allophysis & $+_{b}$ & $\mathbf{C r} \mathbf{r}_{b}$ & $-{ }_{b}$ & $-{ }_{b}$ & $-{ }_{b}$ & $--b$ & $-{ }_{b}$ & $-{ }_{b}$ & 3 & $\mathbf{R}_{\mathrm{b}}$ & $\mathbf{S}_{\mathrm{b}}$ \\
\hline Ethmolaimus pratensis & $-{ }_{b}$ & $\mathbf{C a} \mathbf{a}_{b}$ & $-{ }_{b}$ & $+_{b}$ & $+_{b}$ & $+_{\mathrm{b}}$ & $-{ }_{b}$ & $+{ }_{b}$ & $\mathbf{1}_{\mathrm{b}}$ & $\mathbf{M}_{\mathrm{b}}$ & $\mathbf{S}_{\mathrm{b}}$ \\
\hline Monhystera paludicola & $-{ }_{b}$ & $\mathbf{C a} a_{b}$ & $+_{b}$ & $-{ }_{b}$ & $+_{b}$ & $-{ }_{b}$ & $-b_{b}$ & $-{ }_{b}$ & $2 b_{b}$ & $\mathbf{P}_{\mathrm{b}}$ & $\mathbf{A}_{\mathrm{b}}$ \\
\hline Tripyla glomerans & $+_{b}$ & $\mathbf{C r} \mathbf{r}_{b}$ & $-{ }_{b}$ & $-{ }_{b}$ & $+_{b}$ & $\boldsymbol{t}_{\mathrm{b}}$ & - & $+{ }_{b}$ & $1_{b}$ & $\mathbf{M}_{\mathrm{b}}$ & $\mathbf{S}_{\mathrm{b}}$ \\
\hline Plectus parietinus & + & $\mathbf{C} \mathbf{r}_{b}$ & - & $+{ }_{b}$ & $-{ }_{b}$ & - & $-{ }_{b}$ & -- & $\mathbf{1}_{\mathrm{b}}$ & $\mathbf{P}_{\mathrm{b}}$ & $\mathbf{A}_{\mathrm{b}}$ \\
\hline Eumonhystera barbata & $-{ }_{b}$ & $\mathbf{C a}{ }_{b}$ & $+t_{b}$ & $-{ }_{b}$ & $-{ }_{b}$ & $-b$ & $-{ }_{b}$ & $-b$ & $4_{b}$ & $\mathbf{R}_{\mathrm{b}}$ & $\mathbf{A}_{\mathrm{b}}$ \\
\hline Paractinolaimus macrolaimus & $-b$ & $\mathrm{Cr}_{\mathrm{b}}$ & $+_{b}$ & $+{ }_{b}$ & $+{ }_{b}$ & $+{ }_{b}$ & $-{ }_{b}$ & $+{ }_{b}$ & $\mathbf{1}_{\mathrm{b}}$ & $\mathbf{M}_{\mathrm{b}}$ & $\mathbf{A}_{\mathrm{b}}$ \\
\hline Tylencholaimus minimus & $+_{b}$ & $\mathrm{Cr}_{\mathrm{b}}$ & $-{ }_{b}$ & $-{ }_{b}$ & $-{ }_{b}$ & $-b$ & $-{ }_{b}$ & $-{ }_{b}$ & $3_{\mathrm{b}}$ & $\mathbf{P}_{\mathrm{b}}$ & $\mathbf{A}_{\mathrm{b}}$ \\
\hline Mononchus truncatus & + & $\mathrm{Cr}$ & - & + & + & - & - & - & 3 & $\mathbf{R}$ & $\mathbf{A}$ \\
\hline Achromadora terricola & - & $\mathrm{Cr}$ & - & + & - & + & - & + & 1 & $\mathbf{M}$ & $\mathbf{S}$ \\
\hline Epidorylaimus pseudoagilis & + & $\mathrm{Cr}$ & -- & - & - & -- & + & -- & 3 & $\mathbf{P}$ & $\mathbf{A}$ \\
\hline Plectus aquatilis & ++ & $\mathrm{Cr}$ & - & + & - & -- & - & - & 3 & $\mathbf{M}$ & $\mathbf{S}$ \\
\hline Plectus acuminatus & + & $\mathrm{Cr}$ & - & ++ & - & + & - & -- & 3 & $\mathbf{R}$ & $\mathbf{S}$ \\
\hline Tobrilus zakopanensis & ++ & $\mathrm{Cr}$ & $-{ }_{b}$ & $+_{b}$ & - & $-{ }_{z}$ & $-{ }_{b}$ & $-b$ & $2_{b}$ & $\mathbf{M}$ & $\mathbf{S}$ \\
\hline Prismatolaimus intermedius & -- & $\mathrm{Ca}$ & + & - & + & + & - & + & 1 & $\mathbf{P}$ & $\mathbf{A}$ \\
\hline Eudorylaimus sp.1 & - & $\mathrm{Cr}$ & - & + & ++ & + & + & + & 1 & $\mathbf{R}$ & $\mathbf{S}$ \\
\hline Prionchulus muscorum & ++ & $\mathrm{Cr}$ & - & + & - & -- & - & - & 1 & $\mathbf{M}$ & $\mathbf{S}$ \\
\hline Eumonhystera similis & $+_{z}$ & $\mathbf{C r} \mathbf{r}_{z}$ & $--_{z}$ & ++ & $+_{b}$ & $--_{z}$ & $-{ }_{b}$ & $--_{z}$ & $2_{z}$ & $\mathbf{R}$ & $\mathbf{S}_{\mathrm{z}}$ \\
\hline Ironus paramacramphis & - & $\mathrm{Cr}$ & $-{ }_{b}$ & $-{ }_{b}$ & - & - & $-{ }_{b}$ & ++ & 2 & $\mathbf{R}$ & $\mathbf{S}$ \\
\hline Trischistoma monohystera & -- & $\mathrm{Ca}$ & $+_{b}$ & $+_{b}$ & ++ & + & $-b$ & ++ & 3 & $\mathbf{R}$ & $\mathbf{S}_{\mathrm{z}}$ \\
\hline Prismatolaimus dolichurus & $-{ }_{b}$ & $\mathbf{C a} \mathbf{a}_{b}$ & $+_{b}$ & ++ & $+_{b}$ & $+_{b}$ & - & $+{ }_{b}$ & 2 b & $\mathbf{M}$ & $\mathbf{S}_{\mathrm{b}}$ \\
\hline Aphanolaimus aquaticus & -- & Ca & + & - & + & + & - & ++ & 1 & $\mathbf{P}$ & $\mathbf{A}$ \\
\hline
\end{tabular}

E in cold $(r=-0.20, P<0.01)$ waters. Abundance of guilds $\mathrm{B}$ and $\mathrm{C}$ was not significantly affected by temperature $(P \geq 0.27)$.

\section{DISCUSSION}

In this large scale survey of 94 springs with different environmental conditions, we found on average about 9 nematode species per spring, a number consistent with other surveys of freshwater nematode communities (Traunspurger 2002). Visual inspection of the species accumulation curve (not shown) and Chao's estimate of total number of species suggested that Trentino's springs may host larger numbers of nematode species, some of which remained undetected in our survey. However, analyses of $\beta$ diversity suggest a generally low replacement rate of species along altitudinal gradients, with peaks of the Whittaker index indicating high species turnover approximately where altitudinal changes in vegetation occur in the study region, suggesting that high species turnover in crenic nematode communities may be related to vegetation and climatic zone boundaries.

Models relating community composition to environmental variables showed that the major ecological determinants influencing nematode community composition are water temperature and lithology. This result was consistently obtained from our analyses of different data subsets and is in general agreement with other studies on the ecological determinants of both macroin- vertebrates and meiofaunal diversity patterns, at different spatial scales (Lindegaard et al. 1998, Stoch et al. 2010, this issue). For example, Stoch et al. (2010, this issue) found that the major determinants of diversity patterns in Anellida, Acari and Crustacea, in the same set of springs, were altitude, which is in turn correlated with water temperature, and water chemistry, which is strongly affected by lithology (see also below). Temperature is usually considered an important abiotic parameter influencing benthic community composition, but conflicting published data limit our understanding of the ecological role of this factor in shaping nematode communities. Indeed, other studies have found maximum abundances of freshwater nematodes in different seasons (Traunspurger 2002). Two annual abundance patterns are known for freshwater nematodes, one with a single peak, often a summer maximum, and one with two peaks, in different seasons (Eyualem-Abebe 2006). Univariate analyses also showed that the abundance of three species (Aphanolaimus aquaticus, Ironus paramacramphis and Trischistoma monohystera) increased, while the abundance of five (Epidorylaimus pseudoagilis, Eumonhystera filiformis-vulgaris, E. similis, Plectus acuminatus, P. parietinus) decreased significantly with water temperature (Tab. 5). This pattern is difficult to explain in detail, but agrees with the fact that nematodes of the genus Plectus are often dominant in cold habitats (Procter 1984). These results also confirm that different species have different temperature 
requirements, but again detailed interpretation of the results is hampered by our poor general knowledge of the ecological niches of freshwater nematodes. Indeed, the only general published pattern in the relationships between nematodes and water temperature seems to be that high temperatures $\left(>30{ }^{\circ} \mathrm{C}\right)$ are correlated with small size $(<1 \mathrm{~mm})$ (Ocaña 1991). We note, however, that the temperatures of all the springs included in our study are far lower. The results of the present study may therefore help fill this gap as we observed that, with the exception of E. pseudoagilis, all the species whose abundance decreased with temperature have low c-p values (i.e. 2), while those increasing with temperature have $\mathrm{c}-\mathrm{p}=3$ or 4 . This pattern of variation also appeared in the MI analyses of each spring; c-p increased with water temperature. All this suggests that species replace each other along the crenic water temperature gradient, with $K$-species replacing $r$-species in warm springs. At the same time springs at different temperatures host communities of approximately the same diversity, as indicated by the non-significant regression of diversity indices on water temperature.

However, this is not the only possible interpretation, as water temperature was negatively correlated with spring altitude (see methods), and therefore the significant relation between MI and water temperature could also be seen as an altitudinal gradient of decreasing sediment maturity. Unfortunately, the high collinearity between these independent variables prevented us from disentangling their independent contributions to the variation in $\mathrm{MI}$ and community composition. In conclusion, we have no proof that temperature is important per se in shaping nematode communities. Rather, it probably affects other variables, such as oxygen solubility and food availability, factors more directly related to nematode life-history traits.

Lithology did not significantly affect the diversity indices or the MI, suggesting that ecological communities on either substratum have the same complexity. Rather its influence on the structure of ecological communities seems to be related to variation in the abundance of common species, as indicated by the significant difference in the abundance of 8 species between substrata (Tab. 5). No firm conclusions could be drawn about the influence of $\mathrm{pH}$ and iron ion concentration on the composition of nematode communities, since their significance was inconsistent among different subsamples (Tab. 4) and abundance of only a few common nematode species varied significantly with these ecological parameters (Tab. 4). Also, our general ignorance of the abiotic requirements of nematode species hampered detailed interpretation of these results, although the lack of variation in diversity indices suggests that each species has a substratum preference, although substrata are also known to affect the chemical composition of spring waters. Differential $\mathrm{Ca}^{2+}$ ion tolerance among nematode species may explain their different abun- dances in springs on the two lithologies, but further detailed investigations are required to demonstrate this.

Aquatic bryophytes, sampled at each spring whenever possible, seem to host different communities to soft sediments in the same spring. This emerged from an analysis that takes variability between springs into account, comparing communities sampled from different micro-habitats in the same spring. The large majority of the 24 most abundant species are less abundant in bryophytes than on other substrata. It is known from the literature (e.g., Caldwell 1981) that free-living (not Tylenchomopha) nematodes inhabiting subaerial bryophytes are not significantly affected by bryophyte species, but by the average humidity of the bryophyte (Zullini 1970). We are certain that algae, bacteria and other microorganisms also influence the nematode community, but such analysis is beyond the scope of the present work (see also Stoch et al. 2010, this issue).

The $4^{\text {th }}$-corner statistic revealed low but significant associations between nematode ecology and environmental features of springs. The positive relationship between water temperature and MI, is explained by the larger abundance of predators in warm waters which, in turn, have high c-p values, probably due to the larger complexity of food webs in warm, low altitude springs. This also explains the negative association between MI and altitude. Variation in guild abundance with water temperature simply reflects the above mentioned association between species and environmental characters. Mean weight of nematode species was negatively correlated with DOC and positively with water $\mathrm{pH}$. Springs rich in DOC may host larger quantities of bacteria, thus favouring bacterial feeders that are, on average, small. The increase in mean size of nematodes with $\mathrm{pH}$ is difficult to explain and may be a by-product of increasing abundance with $\mathrm{pH}$ of the large species, D. stagnalis, and the decrease of the small E. similis and of the medium-sized Epidorylaimus pseudoagilis (Tab. 5).

\section{CONCLUSIONS}

Relationships between nematode communities and environmental parameters are rather difficult to detect because of the wide, and poorly defined ecological spectra of individual species. This led some authors to declare that, "the only consistency in patterns of nematode diversity is the apparent inconsistency" (Hodda et al. 2009). Notwithstanding this, our data provide some additional information on freshwater nematode ecology and show that some environmental features, particularly water temperature and lithology, may influence nematode communities. As the same abiotic factors seem to influence distribution of both macroinvertebrates and meiofauna (Lindegaard et al. 1998; Stoch et al. 2010, this issue), this suggests that nematode distribution conforms to the general meiofaunal pattern. In addition, some species characters, such as their c-p value and trophic level, seem to be related to environmental condi- 
tions. In conclusion, nematodes are good ecological indicators of polluted $v s$ unpolluted waters (Wilson \& Kakouli-Duarte 2009), but they are hardly a tool for differentiating typically unpolluted habitats, such as Alpine springs.

\section{ACKNOWLEDGMENTS}

This investigation was made possible by the CRENODAT Project (Biodiversity assessment and integrity evaluation of springs of Trentino - Italian Alps - and long-term ecological research, 2004-2007), funded by the Scientific Research Service of the Autonomous Province of Trento. Zoobenthos samples were collected and sorted by the "CRENODAT team" of the Limnology and Phycology Section of the Museo Tridentino di Scienze Naturali. Chemical data were made available by Dr. Flavio Corradini (Istituto Agrario di S. Michele all'Adige, Trento). The authors are also grateful to Dr. Marco Cantonati for organization and to Dr. Rossana Agoglitta for the calculation of beta diversity.

\section{REFERENCES}

Altherr, E. 1969. Contribution à la connaissance des nématodes del grottes et des eaux interstitielles de Suisse. Bull. Soc. Vaud. Sc. Nat., 70: 255-267.

Altherr, E. 1974. Nématodes de la nappe phréatique du réseau fluvial de la Saale (Thuringe) II. Limnologica, 9: 81-182.

Altherr, E. 1976. Nématodes des eaux stygorhitrales des Alpes autrichiennes. Rev. suisse Zool., 83: 779-847.

Anderson, M.J. 2001. A new method for non-parametric multivariate analysis of variance. Austral Ecol., 26: 32-46.

Andrássy, I. 1956. Die Rauminhalts- und Gewichtsbestimmung der Fadenwürmer (Nematoden). Acta Zool., 2: 1-15.

Andrássy, I. 1978. Nematoda. In: J. Illies (Ed.), Limnofauna Europaea, G. Fisher Verl., Stuttgart: 98-117.

Bates, D., M. Maechler \& B. Dai. 2008. Ime4: Linear mixedeffects models using S4 classes. R package version 0.999375-28.

Bongers, T. 1990. The maturity index: an ecological measure of environmental disturbance based on nematode species composition. Oecologia, 83: 14-19.

Bongers, T. \& H. Ferris. 1999. Nematode community structure as a bioindicator in environmental monitoring. Trends Ecol. Evol., 14: 224-228.

Caldwell, J.R. 1981. Biomass and respiration of nematode populations in two moss communities at Signy Island, maritime Antarctic. Oikos, 37: 160-166.

Cantonati, M., F. Decet, F. Corradini \& E. Bertuzzi 2007. The significance of chemical and physical factors influencing the ecology of springs, and a case study in the south-eastern Alps (Dolomiti Bellunesi National Park). In: M. Cantonati, E. Bertuzzi \& D. Spitale (Eds), The spring habitat: biota and sampling methods. Monografie del Museo Tridentino di Scienze naturali, 4: 45-76.

Cantonati, M., R. Gereke \& E. Bertuzzi. 2006. Springs of the Alps - sensitive ecosystems to environmental change: from biodiversity assessments to long-term studies. Hydrobiologia, 562: 59-96.

Chao, A. 1987. Estimating the population size for capture-recapture data with unequal catchability. Biometrics, 43, 783-791.

Clarke, K.R \& R.M. Warwick. 1998. A taxonomic distinctness index and its statistical properties. J. Appl. Ecol., 35, 523531 .
Clarke, K.R. \& R.M. Warwick. 1999. The taxonomic distinctness measure of biodiversity: weighting of step lengths between hierarchical levels. Mar. Ecol. Progress Series, 184: 21-29.

Clarke, K.R. \& R.M. Warwick. 2001. A further biodiversity index applicable to species lists: variation in taxonomic distinctness. Mar. Ecol. Progress Series, 216: 265-278.

De Coninck, L. A. 1935. Contribution à la connaissance des nématodes libres du Congo Belge. Rev. Zool. Bot. Afr., 26: 211-326.

De Ley, P., W. Decraemer \& Eyualem-Abebe. 2006. Introduction: summary of present knowledge and research addressing the ecology and taxonomy of freshwater nematodes. In: Eyualem-Abebe, W. Traunspurger \& I. Andrássy (Eds), Freshwater nematodes: ecology and taxonomy. CABI Publ., Wallingford: 3-30.

Dixon, P. 2003. VEGAN, a package of R functions for community ecology. J. Veget. Sci., 14: 927-930.

Doledec, S., D. Chessel, C.J.F. ter Braak \& S. Champely. 1996. Matching species traits to environmental variables: a new three-table ordination method. Environ. Ecol. Stat., 3: 143-166.

Dray, S. \& P. Legendre. 2008. Testing the species traits-environment relationships: the fourth-corner problem revisited. Ecology, 89: 3400-3412.

Excoffier, L., P.E. Smouse \& J.M. Quattro. 1992. Analysis of molecular variance inferred from metric distances among DNA haplotypes: Application to human mitochondrial DNA restriction data. Genetics, 131: 479-491.

Eyualem-Abebe, J. Mees \& A. Coomans. 2001. Nematode comminities of Lake Tana and other inland water bodies of Ethiopia. Hydrobiologia, 462: 41-73.

Eyualem-Abebe, W. Traunspurger \& I. Andrássy (Eds). 2006. Freshwater nematodes: ecology and taxonomy. CABI Publ., Wallingford: $752 \mathrm{pp}$.

Eyualem-Abebe, W. Traunspurger \& I.C. Michiels. 2006. Dynamics of freshwater nematodes: abundance, biomass and diversity. In: Eyualem-Abebe, W. Traunspurger \& I. Andrássy (Eds), Freshwater nematodes: ecology and taxonomy. CABI Publ.: 77-93.

Gagarin, V.G. \& L.B. Lemzina. 1992. Fauna nematod mineralnyc histocnikov Kyrgyzstana. Biologia Vnutrennich Vod: Inform. Biol. L., 94: 51-55.

Hodda, M., L. Peters \& W. Traunspurger. 2009. Nematode diversity in terrestrial, freshwater aquatic and marine systems. In: M.J. Wilson \& T. Kakouli-Duarte (Eds), Nematodes as Environmental Indicators. CABI Publ., Wallingford: 45-93.

Hoeppli, R. \& H.J. Chu. 1932. Free-living nematodes from hot springs in China and Formosa. Hong Kong Nat., Suppl. 1: $15-29$.

Hoeppli, R. 1926. Studies of free-living nematodes from the thermal wayers of Yellowstone Park. Trans. Amer. Microsc. Soc., 45: 234-255.

Legendre, P. \& L. Legendre. 1998. Numerical Ecology. Elsevier, Amsterdam: 583 pp.

Legendre, P. \& M.J. Anderson. 1999. Distance-based redundancy analysis: Testing multispecies responses in multifactorial ecological experiments. Ecological Monographs, 69: 1-24.

Lindegaard, C., K.P. Brodersen, P. Wiberg-Larsen \& J. Skriver 1998. Multivariate analysis of macrofaunal communities in Danish springs and springbrooks. In: Botosaneanu, L. (Ed.) 1998. Studies in crenobiology. The biology of springs and springbrooks. Bachuys Publishers, Leiden: 201-220.

Linstow, O. 1901. Beobachtungen an Helminten des Senckenbergischen naturhistorischen Museums des Breslauer zoologischen Instituts und anderen. Arch. mikrosk. Anat. u. Entwicklungsgesch., 58: 182-198. 
Magurran, A.E. 2004. Measuring Biological Diversity. Blackwell Publ.: 256 pp.

McArdle, B.H. \& M.J. Anderson. 2001. Fitting multivariate models to community data: A comment on distance-based redundancy analysis. Ecology, 82: 290-297.

Meyl, A.H. 1953a. Beiträge zur Kenntnis der Nematodenfauna vulkanisch erhitzter Biotope. I. Z. Morph. Ökol. Tiere, 42: 67-116.

Meyl, A.H. 1953b. Beiträge zur Kenntnis der Nematodenfauna vulkanisch erhitzter Biotope. II. Z. Morph. Ökol. Tiere, 42: 159-208.

Meyl, A.H. 1953c. Beiträge zur Kenntnis der Nematodenfauna vulkanisch erhitzter Biotope. III. Z. Morph. Ökol. Tiere, 42: 421-448.

Meyl, A.H. 1954. Die Fadenwürmer (Nematoda) einiger Salzstellen südőstlich von Braunschweig. Abhandl. Braunschweig. Wissensch. Gesell., 6: 84-106.

Michiels, I.C. \& W. Traunspurger. 2005. Benthic community patterns and the composition of feeding types and reproductive modes in freshwater nematodes. Nematology, 7: 21-36.

Ocaña, A. 1991a. Relationship between nematode species and the physico-chemical characteristics of spring waters. I. Conductivity. Nematol. medit., 19: 19-24.

Ocaña, A. 1991b. Relationship between nematode species and the physico-chemical characteristics of spring waters. II. Temperature. Nematol. medit., 19: 25-28.

Ocaña, A. 1992. Relationship between nematode species and the physico-chemical characteristics of spring waters. III. pH, oxygen and iron. Nematol. medit., 20: 181-186.

Ocaña, A. 1993. Nematode fauna in calcic bicarbonate springs in the province of Granada (Spain). Nematol. medit., 21: 199-206.

Ocaña, A. \& R. Morales. 1992. The influence of water ionic composition on the distribution if nematode species in springs of the province of Granada (Spain). Hydrobiologia, 237: 81-92.

Ocaña, A., R. Peña \& R. Morales. 1986. Distribución de los nemátodos del orden Dorylaimida en determinados manantiales minero-medicinales de la provincia de Granada. Limnética, 2: 189-195.

Oksanen, J., Kindt, R., Legendre, P., O'Hara, B., Simpson, G.L., Solymos, P., Stevens, M.H.H. \& H. Wagner. 2009. Vegan: Community Ecology Package. R package version 1.15-3. http://cran.r-project.org/, http://vegan.r-forge.rproject.org/

Paetzold, D. 1958. Beiträge zur Nematodenfauna mitteldeutscher Salzstellen im Raum von Halle. Wissensch. Ztschr. Martin Luther Univ., 8: 17-48.

Pax, F. \& A. Soós. 1943. Die Nematoden der deutschen Schwefelquellen und Thermen. Arch. Hydrobiol., 40: 123183.

Pax, F. \& A. Soós. 1951. Nematoden aus mitteleuropäischen Mineralquellen. Zool. Anz., Leipzig, 145: 92-100.

Pfannkuche, O. \& H. Thiel. 1988. Sample processing. In: R. P. Higgins \& H. Thiel (Eds), Introduction to the study of meiofauna. Washington D.C., USA, Smithsonian Institution Press: 134-145.

Procter, D.L.C. 1984. Towards a biogeography of free-living soil nematodes. Changing species richness, diversity and densities with changing latitude. J. Biogeogr., 11: 103-117.

R Development Core Team (2008). R: A language and environment for statistical computing. $\mathrm{R}$ Foundation for Statistical Computing, Vienna: 1671 pp.

Schmid, P.E. \& J.M. Schmid-Araya. 2002. Trophic relationships in temporary and permanent freshwater meiofauna. In: S.D. Rundle, A.L. Robertson \& J.M. Schmid-Araya (Eds), Freshwater meiofauna. Leiden, The Netherlands, Backhuys Publ.: 295-319.

Seiml-Buchinger, R. \& W. Traunspurger. 2006. Fadenwürmer (Nematoda). In: R. Gerecke \& H. Franz (Eds), Quellen im Nationalpark Berchtesgaden. Nationalpark Berchtesgaden, Forschungbericht, 51: 104-113.

Seinhorst, J.W. 1959. A rapid method for the transfer of nematodes from fixative to anhydrous glycerin. Nematologica, 4: 67-69.

Stoch, F., R. Gerecke, V. Pieri, G. Rossetti, B. Sambugar. 2011. Exploring species distribution of spring meiofauna (Annelida, Acari, Crustacea) in the south-eastern Alps. $J$. Limnol., 70(Suppl. 1): 65-76.

Traunspurger, W. 2000. The biology and ecology of lotic nematodes. Freshwat. Biol., 44: 29-45.

Traunspurger, W. 2002. Nematoda. In: S.D. Rundle, A.L. Robertson \& J.M. Schmid-Araya (Eds), Freshwater meiofauna, Leiden, The Netherlands, Backhuys Publ.: 63-104.

Verhoeven, J.F.K., K.L. Simonsen \& L.M. McIntyre. 2005. Implementing false discovery rate control: increasing your power. Oikos, 108: 643-647.

Warwick, R.M. \& K.R. Clarke. 1995. New 'biodiversity' measures reveal a decrease in taxonomic distinctness with increasing stress. Marine Ecol. Progress Series, 129: 301305.

Wilson, M.J. \& T. Kakouli-Duarte. 2009. Nematodes as Environmental Indicators. CABI Publ, Wallingford: $326 \mathrm{pp}$.

Wilson, M.V. \& A. Shmida. 1984. Measuring beta diversity with presence-absence data. J. Ecol., 72: 1055-1064.

Whittaker, R.H. 1972. Evolution and measurement of species diversity. Taxon, 21: 213-251.

Yeates, G.W., T. Bongers, R.G.M. De Goede, D.W. Freckman \& S. S. Georgieva. 1993. Feeding habits in soil nematode families and genera - An outline for soil ecologists. $J$. Nematol., 25: 315-331.

Zar, H.R. 1999. Biostatistical Analysis. $4^{\text {th }}$ Edition. Prentice Hall, Englewood Cliffs: 929 pp.

Zullini, A. 1970. I nematodi muscicoli della Val Zebrù (Parco Nazionale dello Stelvio): Istit. Lombardo (Rend. Sc.), 104: 88-137.

Zuur, A.F., E.N. Ieno, N.J. Walker, A.A. Saveliev \& G.M. Smith. 2009. Mixed Effects Models and Extensions in Ecology with R. Springer, New York: 574 pp. 